\title{
Electricity Cost Minimization for Delay-tolerant Basestation Powered by Heterogeneous Energy Source
}

\author{
Qingyong Deng ${ }^{1,2}$, Xueming $\mathrm{Li}^{1}$, Zhetao $\mathrm{Li}^{2}$, Anfeng Liü ${ }^{3}$, Young-june Choi ${ }^{4}$ \\ ${ }^{1}$ School of Information and Communication Engineering, Beijing University of Posts and Telecommunications, \\ Beijing 100876, China \\ [e-mail:dengqingyong@xtu.edu.cn;lixm@bupt.edu.cn] \\ ${ }^{2}$ College of Information Engineering, Xiangtan University, Xiangtan 411105, China \\ [e-mail:Liztchina@hotmail.com] \\ ${ }^{3}$ School of Information Science and Engineering, Central South University, ChangSha 410083 China \\ [e-mail:afengliu@mail.csu.edu.cn] \\ ${ }^{4}$ Department of Software, Ajou University, Suwon, 443749, Korea \\ [e-mail: choiyj@ajou.ac.kr] \\ *Corresponding author: Zhetao Li
}

Received March 21, 2017; revised July 6, 2017; accepted August 20, 2017;

published December 31, 2017

\begin{abstract}
Recently, there are many studies, that considering green wireless cellular networks, have taken the energy consumption of the base station (BS) into consideration. In this work, we first introduce an energy consumption model of multi-mode sharing BS powered by multiple energy sources including renewable energy, local storage and power grid. Then communication load requests of the BS are transformed to energy demand queues, and battery energy level and worst-case delay constraints are considered into the virtual queue to ensure the network QoS when our objective is to minimize the long term electricity cost of BSs. Lyapunov optimization method is applied to work out the optimization objective without knowing the future information of the communication load, real-time electricity market price and renewable energy availability. Finally, linear programming is used, and the corresponding energy efficient scheduling policy is obtained. The performance analysis of our proposed online algorithm based on real-world traces demonstrates that it can greatly reduce one day's electricity cost of individual BS.
\end{abstract}

Keywords: Base station, Lyapunov optimization, queue model, online algorithm, linear programming 


\section{Introduction}

Green communication technology has attracted more attention recently due to the amazing energy consumption for information and communication (ICT), approximate 1,700 TWh in 2030. The amazing energy consumption not only increases $\mathrm{CO}_{2}$ emissions but also leads to substantial costs and heavy loads for power grid. Cellular networks constitute a significant part of the ICT sector and base stations (BSs) have the largest share of the energy consumption of cellular networks. The growing interest in new and reliable services in mobile telecommunications has resulted in an increased number of installed BSs worldwide. As the 5G era arrive, the number of base stations will increase dramatically. However, operators will continue using the existing infrastructure resources in the long term to save the construction cost of BSs and multi mode access will coexist in the already installed BSs. What's more, more and more internet of thing (IOT) services will be carried out through cellular network due to its wide coverage in the future, such as smart meter reading and will add new energy consumption to existing BSs. Therefore, reducing the energy consumption of BSs has recently become an important research topic.

It is well known that the electricity cost has become a significant portion of the operational expenditure of cellular operators, for example the electricity cost of all the national BSs reaches approximately 28 billion per year in China. Therefore, how to reduce the electricity bills attracts more interest for cellular operators. In this paper, we investigate the forthcoming multi-mode sharing base station and exploit the unquie characteristics of cellular IOT service to reduce the electricity bills. To the best of my knowledge, cellular IoT services can be divided into delay-sensitive and delay-tolerant tasks. For example, the smart meter data usually allows several minutes or even hours of delay before reaching the operation center. In order to make cellular IoT "greener", renewable energy is introduced to power cellular BSs in the future and makes the power supply of BSs more flexible. However, renewable energy sources are highly intermittent and uncontrollable in nature. Therefore, the delay-tolerant BS with multiple energy sources including power grid, battery storage, and local solar/wind renewable energy is considerd in this paper to reduce electricity costs.

The rest of this paper is organized as follows: Section 2 presents an overview of the most promising approaches dedicated to improving the energy-efficiency of the cellular networks and BSs. Section 3 presents our system models, Section 4 analyzes the performance of our proposed method, and Section 5 gives the conclusion and the directions of future work.

\section{Related Work}

Many existing studies aim at improving energy efficiency in the cellular access networks and can be concluded in three ways: resource allocation, dynamic management of network resources and energy harvest. A lot of research focuses on improving energy efficiency through resource allocation optimization. In order to maximize the energy efficiency of OFDMA-based transmissions, both subchannel and power allocation were considered in [1]. Then a joint optimization model of Quality of Experience and power consumption in OFDMA multi-cell networks was proposed in [2]. An iterative power control algorithm and a near optimal sensing time scheme were developed in [3]. From the point of network level, one of the most important approaches for reducing energy consumption is dynamic management of 
network resources. For the purpose of minimizing long-term energy cost in cellular networks, dynamic BS activation and transmission power control were considered in [4], but limited their attention to distributed solutions. Bu et al. [5]discussed how the dynamic operation of cellular BSs affect the traffic, realtime electricity price, and the pollutant level associated with electricity generation. Coordinated multipoint was used to ensure acceptable service quality in the cells and the active BSs decided on which retailers to procure electricity from and how much electricity to procure. A joint optimization method considering both partial spectrum reuse scheme and base-station-sleep was proposed for improving the energy efficiency for heterogeneous networks in [6]. Another effective aspect of reducing energy consumption is energy harvest. In industry wireless sensor network, energy harvesting model of a node to adjust transmission power was considered in [7], where in ultra dense networks, an effective interference coordination mechanism is proposed to cognitively limit the interference between the BSs and users in [8]. Joint resource allocation and energy harvest in distributed antenna was described in [9]. An iterative method for power allocation and subchannel assignment was proposed in energy harvesting enabled heterogeneous cloud small cell network [10]. Works mentioned above often solve the problem from the angle of network and need the cooperation of BSs and users, while energy allocation is not considered. Most of the works are concerned about energy efficiency of cellular network and few works about energy efficiency of individual BS. In this paper, the stochastic characteristics of renewable energy and load demand will be carefully studied. It is well known that the technique of Lyapunov optimization is initially developed for dynamic control of queuing systems for wireless networks, but many researchers utilize the Lyapunov optimization technique to solve the scheduling problem, such as energy allocation, which does not require prior statistical information and is provably close to optimal. Yu et al. [11]took the electricity cost minimization problem into consideration in a residential micro-grid consisting of multiple households equipped with multiple energy resources. Efficient algorithms for energy dispatch were studied via Lyapunov optimization techniques, which have also been used in the electric vehicle charging scenario with intermittent renewable energy sources in [12]. Josip et al. in [13] performed an investigation regarding the impact of the traffic intensity on the power consumption of BSs through analyzing five days' energy consumption of a real indoor BS site containing BSs of GSM 900, GSM 1800 and UMTS access technologies and confirmed that the instantaneous power consumption of BSs varies in accordance with the traffic load. These are precisely the research premises of our study. When should the delay-tolerant tasks start and how much electricity should be procured from power grid to ensure them finished before deadline are considered in this paper and an online energy and traffic scheduling strategy is designed to reduce the electricity cost in accordance with the current traffic load and energy supply level. We introduce the queue theroy to model the energy demand of each access mode and multiple queues model is established to ensure the constraints, such as worst delay and battery level, then Lyapunov optimization method is applied to work out the online scheduling policy and result in lowest electricity cost for cellular operators.

Our main contributions can be summarized as follows:

(1) A multiple queueing model for multi-mode sharing BS is proposed considering energy sources and QoS of users.

(2) A smart basestation is presented and Lyapunov optimization is used to solve the energy dispatch problem aiming to minimize the electricity cost, and then realworld traces simulation is performed to verify our proposed online algorithm with appropriate settings. A salient feature of our algorithm is that it requires no piror information of the system and can be easily implemented online. 


\section{System Models}

We consider a multi-mode sharing BS equipped with multiple energy resources as shown in Fig. 1. We assume that the multiple energy supplies of the BS are in the form of interconnection, the renewable power sources are located close to BSs and only operation cost is taken into consideration, thus we only need to consider the electricity cost procured from the power grid.

\subsection{Energy Consumption Model for BS}

To reduce the energy consumption of cellular networks, precise knowledge about BS energy consumption and the influence of the traffic load on the instantaneous BS power consumption can be of great importance. As is mentioned in [13], the elements of a BS can be divided into two groups: radiofrequency equipment and support system. Radiofrequency equipment includes power amplifiers and transceivers that are used to serve one or more sectors/cells, while support system includes alternate current/direct current (AC/DC) power conversion modules, air conditioning elements, analogue and digital signal processors, etc. In order to facilitate analysis, we denote the energy consumption of BS as $P_{B}(t)$ and express it as follows:

$$
P_{B}(t)=P_{b x}(t)+P_{m i s c}(t)
$$

where

$$
P_{t x}(t)=P_{\alpha} * \mu(t)+P_{\beta}
$$

where $P_{t x}(t)$ is the instantaneous BS power consumption, which is dynamic and has been confirmed to have linear correlation with the traffic load in [7], and $\mu(t)$ is the traffic load factor of each access mode, $P_{\alpha}$ is a traffic dependent part, $P_{\beta}$ is the base cost for activating a base station. $P_{\text {misc }}(t)$ denotes the constant part of energy consumption such as air condition, etc.

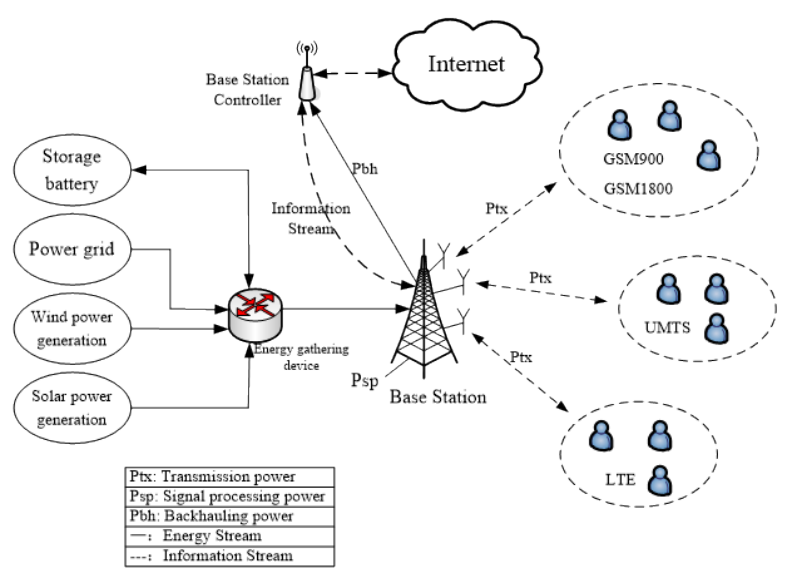

(a) system model 


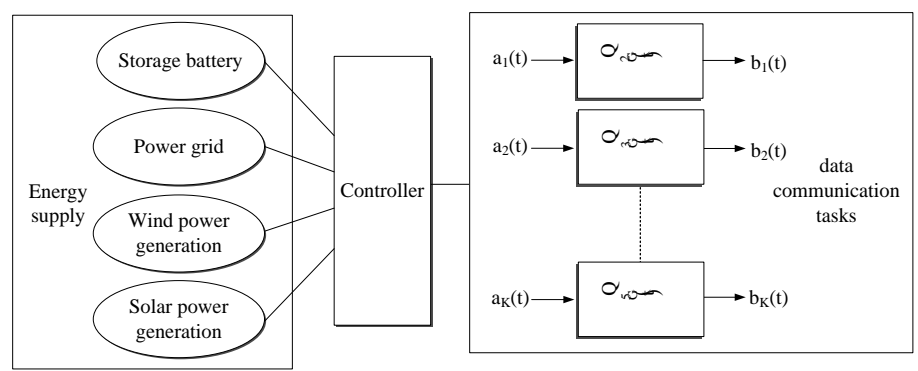

(b) scheduling model

Fig. 1. Multi-mode sharing BS model

\section{2 Queue Model}

In cellular IoT network, massive wireless sensor data are delivered to server application through BS, and can be divided into two types according to service requirement: delay-sensitive and delay-tolerant, corresponding to non-deferrable and deferrable communication tasks. A suitable percentage of deferrable tasks will be studied in the following simulation. The communication rate of deferrable task can be controlled within an acceptable range due to QoS of network users. Therefore, the demand of communication task queue model for BS can be transformed into the queue of energy demand on the basis of (2). We formulate our problem based on a queuing model [14] and propose an online energy dispatch algorithm without knowing any unpredictable variables, such as renewable energy output and electricity price.

In this work, we assume that the communication tasks can be achieved during $T$ timeslots, which can be divided into multiple timeslot $t, t=\{1,2, \cdots T\}$.The renewable energy output is time variant, unpredictable and denotes as $s(t)$ at timeslot $t$. During every timeslot $t$, the amount of energy demand is $W(t)$, the sum of deferrable energy demand is $w_{d}(t)$ and non-deferrable energy demand is $w_{u}(t)$, as shown in equation (3).

$$
\begin{aligned}
& W(t)=w_{d}(t)+w_{u}(t), \\
& w_{d}(t) \geq 0, w_{u}(t) \geq 0 .
\end{aligned}
$$

During the timeslot $t$, the amount of energy consumption can be denoted as $Y(t)$, including the energy dispatched to deferrable $x(t)$ and non-deferrable $w_{u}(t)$. To ensure the accomplishment of non-deferrable communication tasks, we have $L(t) \geq w_{u}(t)$. Then we get $L(t)$ in equation (4) as follows:

$$
L(t)=x(t)+w_{u}(t)
$$

It is universally acknowledged that data will be stored in a queue and served on a First-In-First-Out (FIFO) basis in the BS, therefore, we can formulate the data queue into energy demand queue in general without considering channel conditions. In this paper, we denote $Q(t)$ as the total deferrable tasks in timeslot $t$ in a single queue, and the queue backlog growth follows:

$$
Q(t+1)=\max [Q(t)-x(t), 0]+w_{d}(t) .
$$


Due to coexistence of GSM900/1800, UMTS and LTE in current multi-mode sharing BS, single-queue equation can be modified into evolving equation of $G$ queues as follows:

$$
Q_{g}(t+1)=\max \left[Q_{g}(t)-x_{g}(t), 0\right]+w_{d, g}(t) ; \forall g, t,
$$

where $Q_{g}(t), x_{g}(t)$ and $w_{d, g}(t)$ denote queue backlog, energy distribution and energy demand for deferrable tasks in timeslot $t$ of each queue $g$. As shown in Eqn. (7).

$$
Q(t)=\sum_{g=1}^{G} Q_{g}(t), x(t)=\sum_{g=1}^{G} x_{g}(t), w_{d}(t)=\sum_{g=1}^{G} w_{d, g}(t) .
$$

\section{3 Battery Model}

UPS batteries in BSs are usually deployed for emergency situations, such as power outage. In the aforementioned BS model, it is considered as energy storage in the power supply system and can discharge when the electricity price is high to reduce the amount of electricity procured from the grid. We denote the battery capacity as $E_{\max }$, the necessary energy level of battery in emergency situation as $E_{\min }$ and the current energy level of the battery as $E(t)$ respectively. The battery energy level should always be non-negative and can not exceed the battery capacity. We consider the battery charge from power grid rather than renewable resource because of the intermittence of renewable energy. The power charged to (when $p_{e}(t)<0$ ) or discharged from (when $p_{e}(t)>0$ ) the battery during period $t$ is denoted as $p_{e}(t)$, as follows:

$$
\begin{aligned}
& E(t+1)=E(t)-p_{e}(t), \\
& E_{\min }<E(t)<E_{\text {max }} ; \forall t, \\
& -\left[E_{\max }-E(t)\right] \leq p_{e}(t) \leq E(t) .
\end{aligned}
$$

As different batteries allow different maximum charging/discharging rates, we donote $B_{c}$ as the maximum charging rate and $B_{d}$ as the maximum discharging rate per timeslot. We can get another constraint condition to $p_{e}(t)$ as

$$
\max \left\{-B_{c},-\left[E_{\max }-E(t)\right]\right\} \leq p_{e}(t) \leq \min \left(B_{d}, E(t)-E_{\min }\right) .
$$

\section{Energy Efficient Scheduling}

The BS needs to procure energy from the smart grid and we denote it as $\left[L(t)-s(t)-p_{e}(t)\right]^{+}$ and real-time electricity price as $e(t)$. The following constraints are made to bound the values of $w_{d}(t)$ and $x(t)$ :

$$
0 \leq w_{d}(t) \leq w_{d, \max }, 0 \leq x(t) \leq x_{\max } ; \forall t,
$$


where $w_{d \text {,max }}$ is the maximum deferrable energy demand and $x_{\max }$ is the maximum energy distribution for deferrable tasks per timeslot. We further assume $x_{\max } \geq w_{d, \max }$ to ensure the stability of queue.

Our objective is to minimize the time average electricity cost for individual multi-mode sharing $\mathrm{BS}$ in the long run, as $\mathrm{P} 1$ :

$$
\min _{x_{g}(t), p_{e}(t)} \lim _{t \rightarrow \infty} \frac{1}{t} \sum_{\tau=0}^{T-1} \mathbb{E}\left\{e(\tau)\left\{\max \left[L(\tau)-s(\tau)-p_{e}(\tau), 0\right]\right\}\right\}
$$

subject to:

$$
\begin{aligned}
& \bar{Q}_{g}<\infty ; \forall g, \\
& 0 \leq x(\tau) \leq x_{\max } ; \forall \tau, \\
& \overline{\frac{Q_{g}(\tau)}{R_{g}}+w_{d, g}(\tau)-x_{g}(\tau)} \leq 0, \forall g, \tau,
\end{aligned}
$$

where constraint (12) guarantees that all the queues are stable, constraint (13) sets a limit on the maximum energy distribution of deferrable demand. We denote $\bar{Q}_{g}$ as time-average energy demand backlog of $g$, and $\bar{Q}_{g}$ is mathematically defined in Eqn. (15).

$$
\bar{Q}_{\mathrm{g}} \triangleq \lim _{T \rightarrow \infty} \sup \frac{1}{T} \sum_{t=0}^{T} E\left\{Q_{g}(t)\right\}
$$

We rewrite (14) into the following inequality:

$$
\frac{\overline{Q_{g}(\tau)}}{\overline{x_{g}(\tau)-w_{d, g}(\tau)}} \leq R_{g} ; \forall g, \tau
$$

Where $\overline{x_{g}(\tau)-w_{d, g}(\tau)}$ denotes average queue processing rate, and $R_{g}$ is the queue processing time. Since there are $G$ types of access mode, each with acceptable processing time $R_{g}, g \in\{1,2, \ldots, G\}$ as discussed in [12]. With the rapid development of access technology, energy efficiency and processing rate also have been improved, therefore, $R_{g}(L T E)<R_{g}(U M T S)<R_{g}(G S M)$. The Inequality (16) imposes limits on the average processing time of deferrable tasks.

\subsection{Delay-Aware Virtual Queue}

Note that (11) does not include the terms accounting for delay constraints. In order to make the function delay-aware, the virtual queues [12] are applied and defined as $Z_{g}(0)=0 ; \forall g$ and

$$
Z_{g}(t+1)=\max \left\{Z_{g}(t)+\frac{\eta}{R_{g}} 1_{Q_{g}(t)>0}-x_{g}(t), 0\right\} ; \forall g, t
$$


We can see from (17), $\frac{\eta}{R} 1_{Q_{g}(t)>0}$ imposes a penalty to the virtual queue backlog when there is unserved task in the actual queue by ensuring that $Z_{g}(t)$ grows whenever the actual queue backlog is not empty. $1_{Q_{g}(t)>0}$ is an indicator function that equals to 1 when $\left(Q_{g}(t)>0\right)$ and 0 else. The growth rate of the virtual queue can be controlled by $\eta$, which ensures that the queue has a worst case (longest) processing delay $\delta_{\max }$. Assuming the queue $Q_{g}(t)$ and queue $Z_{g}(t)$ have finite upper bounds $Q_{g, \max }$ and $Z_{g, \max }$. So $\delta_{\max }$ can be defined as [15].

$$
\delta_{\text {max }} \triangleq\left[\frac{\left(Q_{g, \text { max }}+Z_{g, \text { max }}\right) R_{g}}{\eta}\right]
$$

\subsection{Lyapunov Optimization}

We propose Lyapunov optimization method to solve P1 in this work. Unlike dynamic programming method usually has the problem of "curse of dimensionality", Lyapunov optimization method [16] provides a simple online solution only based on the current knowledge of the system state. Considering the fluctuant character of communication load, real-time property of electricity price $e(t),\left\{e_{\min } \leq e(t) \leq e_{\max }\right\}$, and intermittent feature of green energy $s(t)$, Lyapunov optimization method is suitable to solve P1 without any future information about communication load, electricity price or green energy availability. Before doing so, we have to note that Lyapunov optimization can not be directly applied to solve our problem due to the presence of battery constraint (8), which couples the decisions across timeslots. We introduce a virtual queue $H(t)$ to represent the battery energy level. To relax the constraint of battery, the virtual battery queue [17] will be transformed to another form as shown in Eqn. (19).

$$
H(t)=E(t)-V e_{\max }-B_{d}-E_{\min }
$$

where $V$ is a tradeoff between objective and queue backlog growth and can also be used to constrain battery capacity. Lyapunov function is derived in consideration of the aforementioned queues ( $Z(t), H(t), Q(t))$ as Eqn. (20).

$$
\psi(\Theta(t))=\frac{1}{2} \sum_{g=1}^{G}\left[Z_{g}^{2}(t)+Q_{g}^{2}(t)\right]+\frac{1}{2} H^{2}(t)
$$

Then the conditional Lyapunov drift can be described as

$$
\Delta(\Theta(t)) \triangleq \mathrm{E}\{\psi(\Theta(t+1))-\psi(\Theta(t)) \mid \Theta(t)\}
$$

where $\Theta(t)$ is the state vector $\Theta(t) \triangleq(Z(t), Q(t), H(t))$. In consideration of both the electricity cost and queue backlog growth, our objective is to minimize the following function in each timeslot $t$, Eqn. (22).

$$
\min _{x_{g}(t), p_{e}(t)}\left\{\Delta(\Theta(t))+V E\left\{e(t)\left\{\max \left[L(t)-s(t)-p_{e}(t), 0\right]\right\} \mid \Theta(t)\right\}, \forall t\right.
$$


where the left part is the growth of the queue and the right part is the expected electricity cost for the communication load of the BS. The objective is to minimize the weighted sum of drift and penalty (cost), which can be proven that it holds. The following inequality holds:

$$
\begin{aligned}
& \Delta(\Theta(t))+V \mathrm{E}\left\{e(t)\left\{\max \left[L(t)-s(t)-p_{e}(t), 0\right]\right\} \mid \Theta(t)\right\} \\
& \leq C+V \mathrm{E}\left\{e(t)\left\{\max \left[L(t)-s(t)-p_{e}(t), 0\right]\right\} \mid \Theta_{t}\right\}+\sum_{g=1}^{G} Q_{g}(t) \mathrm{E}\left\{\left[w_{d, g}(t)-x_{g}(t) \mid \Theta_{t}\right\}\right. \\
& +\sum_{g=1}^{G} Z_{g}(t) \mathrm{E}\left\{\left[\frac{\eta}{R_{g}}-x_{g}(t) \mid \Theta_{t}\right\}-\mathrm{E}\left\{X(t) p_{e}(t) \mid \Theta_{t}\right\}\right.
\end{aligned}
$$

where $C$ is defined as:

$$
C=\frac{\sum_{g=1}^{G}\left[x_{g, \text { max }}^{2}+w_{d, g, \max }^{2}\right]}{2}+\frac{\sum_{g=1}^{G} \max \left[\left(\frac{\eta}{R_{g}}\right)^{2}, w_{d, \text { max }}^{2}\right]}{2}+\frac{\max \left[B_{c}^{2}, B_{d}^{2}\right]}{2}
$$

The detailed derivation steps can be found in [17].

\subsection{Online Optimization Algorithm}

Since $C$ is a constant, our objective can be considered as minimizing the right-hand side of (23) and can be transformed to an online optimization algorithm as algorithm1.

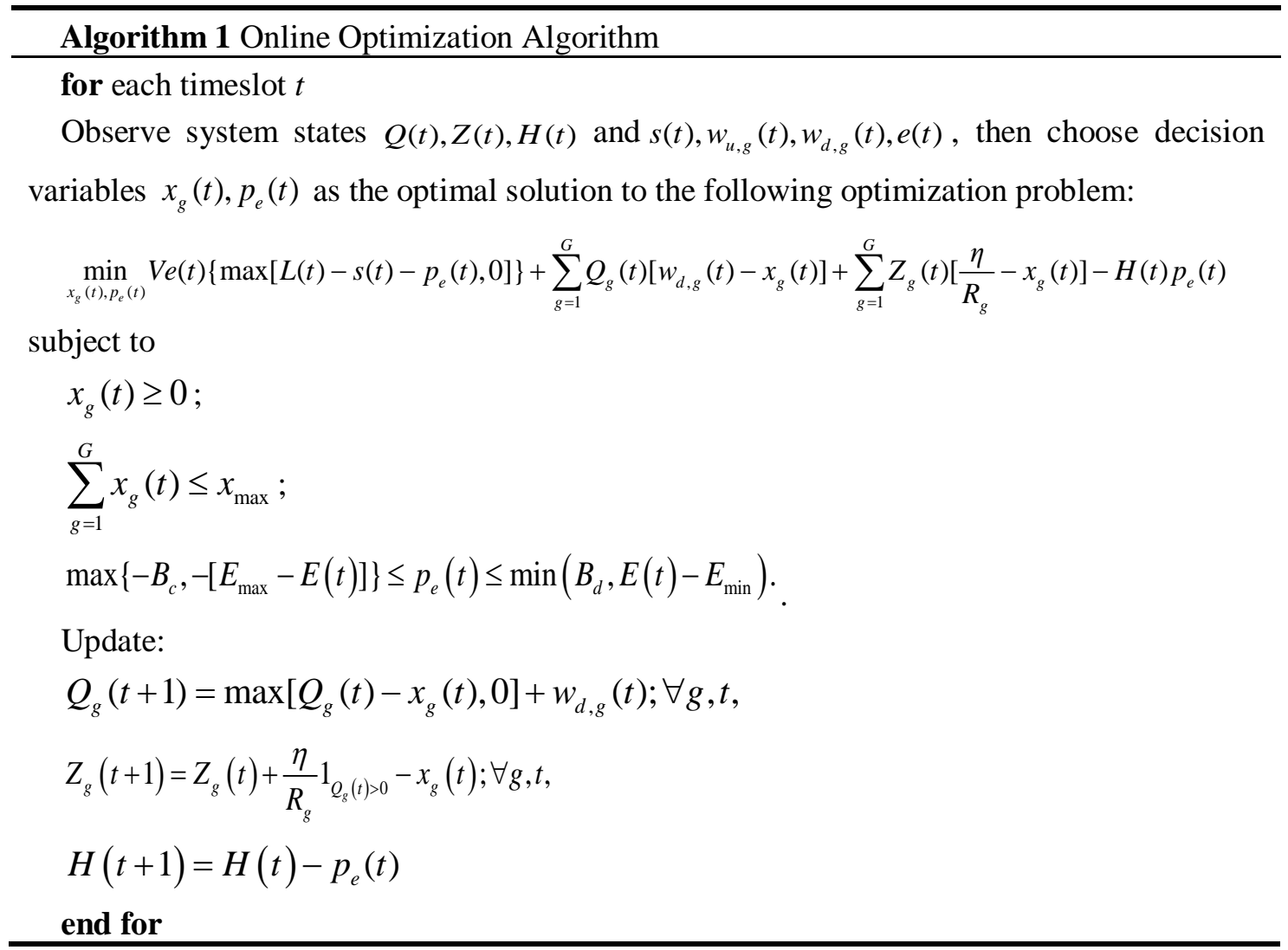




\subsection{Dynamic Algorithm Solution}

Therefore, optimization problem is converted to an easier form P2:

$$
\begin{gathered}
\min _{x_{g}(t), p_{e}(t)} \sum_{g=1}^{G}\left[V e(t)-Q_{g}(t)-Z_{g}(t)\right] x(t)-[\operatorname{Ve}(t)+H(t)] p_{e}(t), \\
\text { s.t. } \quad\left\{\begin{array}{c}
0 \leq x_{g}(t) \leq x_{\max }, \sum_{g=1}^{G} x_{g}(t) \leq x_{\max } ; \forall g, \\
L(t)-s(t)-p_{e}(t)>0 .
\end{array}\right.
\end{gathered}
$$

Then $x_{g}(t)$ and $p_{e}(t)$ can be obtained from the solution of $\mathrm{P} 2$ at the beginning of each timeslot $t$. In order to solve P2, we should define the upper bound of $\mathrm{V}$ at first. From the aforementioned analysis, we can see:

$$
V_{\max }:=\min \frac{E_{\max }-E_{\min }-B_{d}}{e_{\max }-e_{\min }}
$$

Solution: If $H(t)<-V e(t)$, the optimal solution always choose $p_{e}(t) \leq 0$, therefore, the battery should be charged from power grid, $p_{e}(t)=\max \left\{-B_{c},-\left[E_{\max }-E(t)\right]\right\}$;

If $H(t)>-V e(t)$, the optimal solution always choose $p_{e}(t) \geq 0$, therefore, the battery should discharge to BS as much as possible, $p_{e}(t)=\min \left(B_{d}, E(t)-E_{\min }\right)$;

If $Q_{g}(t)+Z_{g}(t)<V e(t)$, the optimal solution is procure less electricity from power grid only to guarantee the non-deferrable task during this timeslot, $x_{g}(t)=0$;

If $Q_{g}(t)+Z_{g}(t)>V e(t)$, the optimal solution is work for deferrable tasks as much as possible in timeslot $t, \sum_{g=1}^{G} x_{g}(t)=x_{\max }$.

The above analysis shows that we can get the scheduling result easily, so the complexity of the proposed online algorithm 1 is negligible and can be easily implemented online.

\section{Experimental Classification Results and Analysis}

In this section, we perform simulations on data sets with 10-minutes timeslot interval. The real-time electricity price used in simulation is the real-time electricity price data of GuangZhou City on July 01, 2013, as Fig. 2.

For simplicity, we employ wind energy as renewable energy source. Real wind speed data are taken from [18] and converted to wind power generation (Fig. 3) with 10-minute granularity, based on the specifications of the $2 \mathrm{~kW}$ wind power generator. 


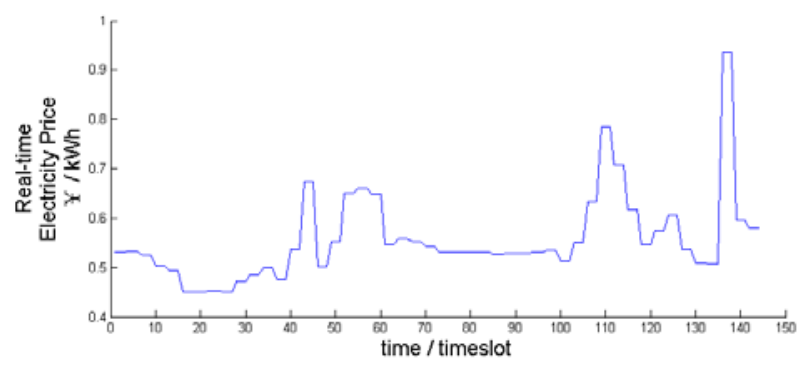

Fig. 2. Real-time electricity price

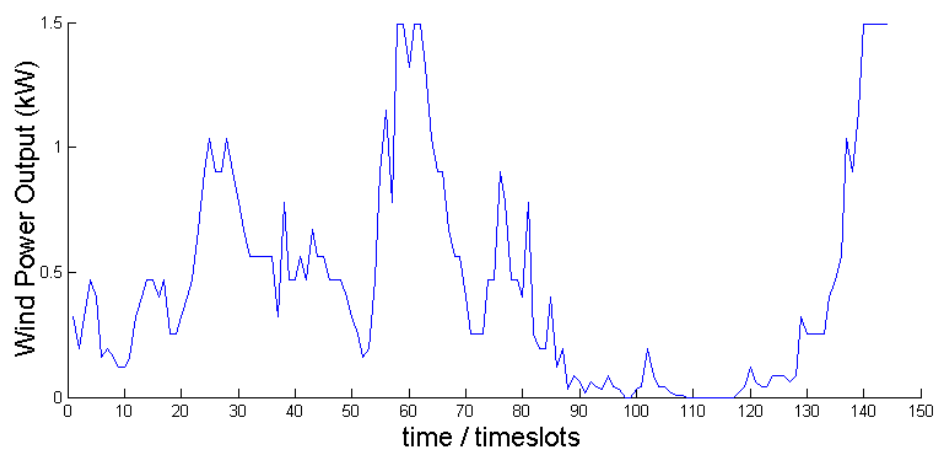

Fig. 3. Wind generation power

In the simulation, 2000 communication tasks are generated for a scheduling period from 12:00 AM to 12:00 AM in the next day, which means 144 timeslots. The increase in user activity during a day results in an increase in the instantaneous power consumption of a BS and vice versa, so the shape of power consumption pattern is a direct consequence of a daily traffic pattern variation. From [13] we know the highest power consumption was recorded between 10:00 and 14:00, 19:00 and 22:00 for each of the analyzed days, while the lowest consumption was observed between 02:00 and 07:00. For a fine-grained reflection of the reallife communication pattern, we adopt normal distributions to model the arrival of communication tasks. Specifically, it follows four normal distributions with the mean of $\mu=7: 30 A M$, $\mu=12 P M, \mu=6 P M$ and $\mu=10 P M$, respectively, and the standard deviation of $\sigma=1$ hour. The Min and Max power consumption per hour are obtained from [13]. The related simulation settings are summarized in Table 1.

Table 1 SIMULATION SETTINGS

\begin{tabular}{c|c}
\hline Total number of timeslots $t$ & 144 \\
\hline Total number of communication tasks & 2000 \\
\hline Maximum charging rate $B_{\mathrm{c}}$ & $240 \mathrm{~W}$ \\
\hline Maximum discharging rate $B_{\mathrm{d}}$ & $240 \mathrm{~W}$ \\
\hline Minimum energy level $E_{\min }$ & $2.88 \mathrm{kWh}$ \\
\hline Battery capacity $E_{\max }$ & $14.4 \mathrm{kWh}$ \\
\hline Queue processing time $\mathrm{R}$ & {$[2,1]$ time slots } \\
\hline Types of access mode $\mathrm{G}$ & $2(\mathrm{GSM}$ and $\mathrm{UMTS})$ \\
\hline Min. power consumption $/ \mathrm{mode}$ & {$[2.4235,0.5419] \mathrm{kWh}$} \\
\hline Max. power consumption /mode & {$[3.877,0.6994] \mathrm{kWh}$} \\
\hline
\end{tabular}




\subsection{Balance Between Electricity Cost and Delay Time}

We studied the impact of parameter $V$ and $\eta$ on the total electricity cost and mean delay time. From the above settings, we compute the upper bound of $\mathrm{V}$ as nearly 250 . In order to study the impact of parameter $\eta$ on the electricity cost and the mean delay time, we show the relationship between electricity cost and the vaule of $\eta$ and the relationship between the mean delay time and the vaule of $\eta$ in Fig. 4. As we can see, the electricity cost increases non-lineary with the vaule of $\eta$, while the mean delay time decreases with the increasing vaule of $\eta$. The electricity cost and the mean delay time reach saturation when $\eta$ is large enough, the total electricity cost is close to its maximum and the mean delay time will reach its minimum, which means deal with the communication tasks upon arrival.

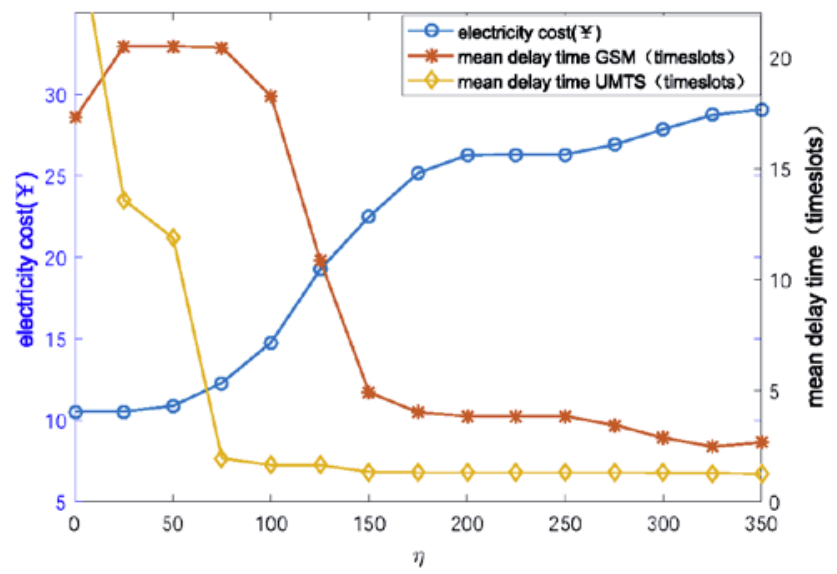

Fig. 4. Total electricity cost and mean delay time vs. the value of $\eta$. The value of $V$ is set to 120 .

Similarly, we can observe the reduction of electricity cost and the increase of mean delay time as $V$ increases in Fig. 5. From the result we can see that with the increasing vaule of $V$, the electricity cost decreases, which can be interpreted by (22). When the vaule of V exceed 250, the electricity cost and the mean delay time no longer change and prove the correctness of our theory analysis. From the above result, we can see that there is a balance between delay time and electricity cost, where $\eta$ is set to 100 and V is set to 120 .

\subsection{Study on Percentage of Deferrable Task}

It is impossible to allow all tasks to delay. As suggested by the result shown in Fig. 6, we can see with the increasing vaule of percentage, the electricity cost decreases but the mean delay time increases. It is suitable when the percentage of deferrable task is set to $20 \%$ to $50 \%$, in order to keep the mean delay time under an acceptable level. The percentage used in the above experiments is set to $35 \%$.

\subsection{Performance on Energy Scheduling}

The simulation result (Fig. 7) shows the energy scheduling policy, which means how much electricity we should get from power grid in each timeslot when the electricity cost and delay time are both suitable. As the Fig. 8 shown, we need to get large amounts of electricity from power grid when there is little wind power output, whereas we only need to get little when there is much renewable energy. Similarly, high real-time electricity price also affects the scheduling policy. We can see that the policy made by the above algorithm is reasonable. 


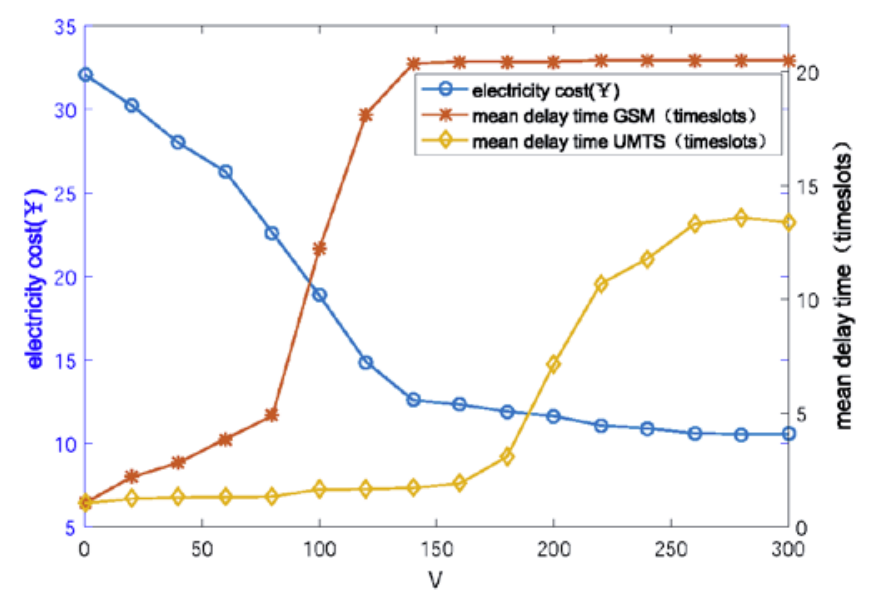

Fig. 5. Total electricity cost and mean delay time vs. the value of $V$. The value of $\eta$ is set to 100 .

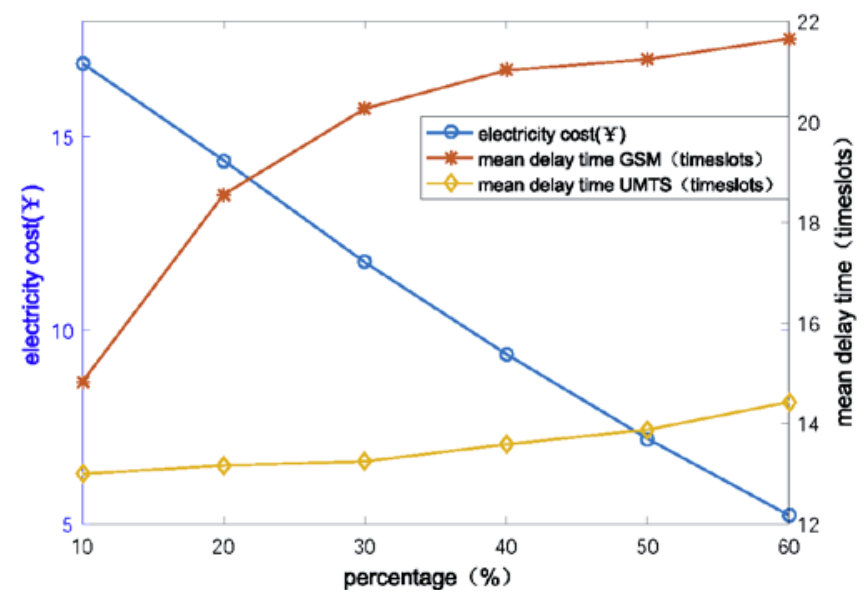

Fig. 6. Total electricity cost and mean delay time vs. the percentage of deferrable task.

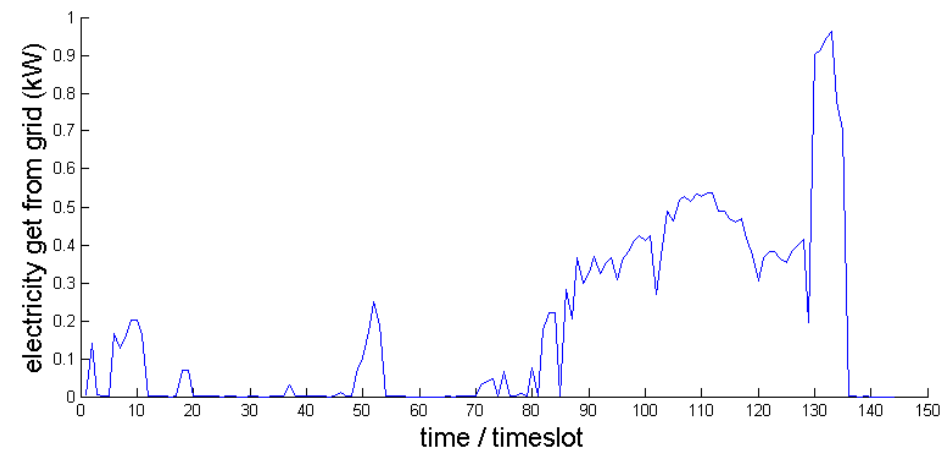

Fig. 7. Electricity procured from grid 


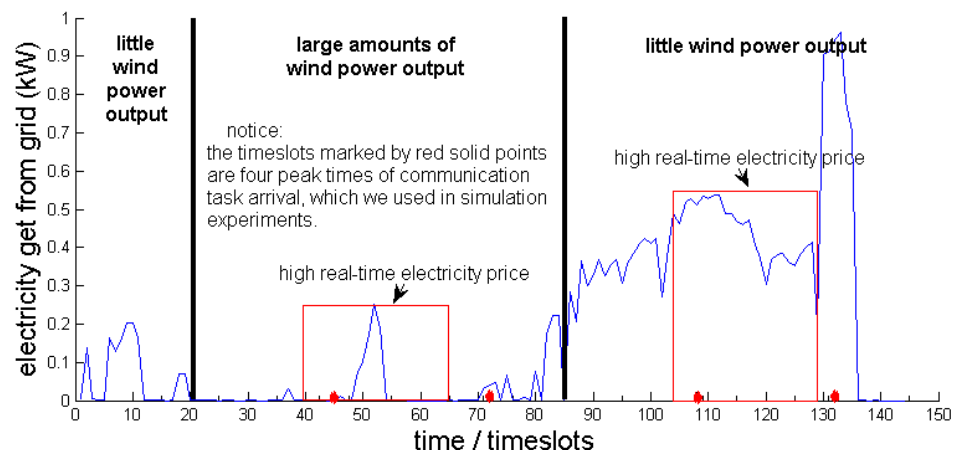

Fig. 8. Analysis of scheduling policy

\subsection{Performance on Electricity Cost}

The result shows that the communication electricity cost of multi-mode BS of one day is 14.8 when using Lyapunov optimization and choosing the appropriate value for parameter $\mathrm{V}$ and $\eta$, while 47.19 without renewable energy and 21.65 without introducing Lyapunov optimization algorithm, which means there is no deferrable task. The algorithm we proposed can reduce the total electricity cost by $68.63 \%$ and $31.64 \%$ respectively. The electricity cost of one day corresponding to different situation is presented in Fig. 9.

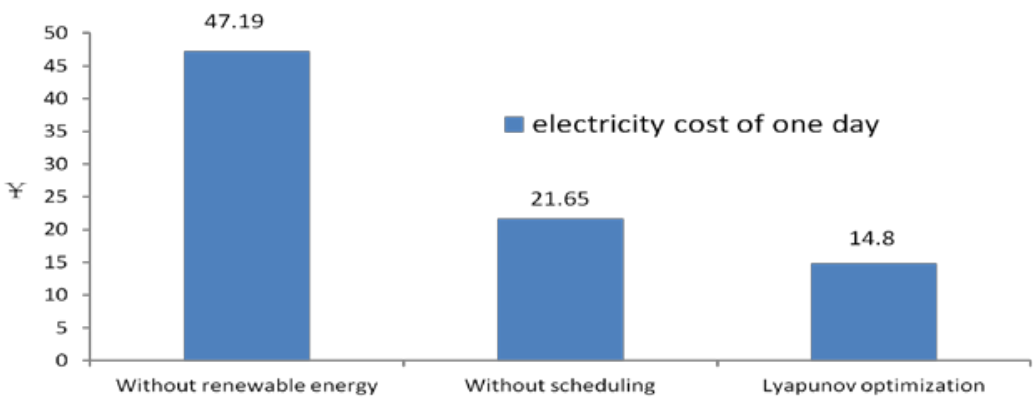

Fig. 9. Comparison of electricity cost with different strategy

\section{Conclusion}

To the best of our knowledge, few studies have been proposed to investigate the cellular BS equipped with multiple energy sources including power grid, renewable energy and local storage. In this work, Lyapunov technique has been used to minimize the electricity cost of the BS. Finally, the solution to the proposed optimal problem is achieved to form the corresponding energy efficient scheduling policy. Simulation results, which are based on real data, show that our proposed online algorithm can greatly reduce the electricity cost of per BS by $68.63 \%$. Future work is in progress to consider how to choose an optimal battery capacity and prolong the lifetime of storage battery, on which the times of charging and discharging have an obvious effect. 


\section{References}

[1] C. Xiong, G. Y. Li, S. Zhang, Y. Chen and S. Xu, "Energy-Efficient Resource Allocation in OFDMA Networks," IEEE Transactions on Communications, vol. 60, no. 12, pp. 3767-3778, Dec. 2012. Article (CrossRef Link).

[2] H. Shao et al., "Joint Optimization of Quality of Experience and Power Consumption in OFDMA Multicell Networks," IEEE Communications Letters, vol. 20, no. 2, pp. 380-383, Feb. 2016. Article (CrossRef Link).

[3] H. Zhang, Y. Nie, J. Cheng, V. C. M. Leung and A. Nallanathan, "Sensing Time Optimization and Power Control for Energy Efficient Cognitive Small Cell With Imperfect Hybrid Spectrum Sensing," IEEE Transactions on Wireless Communications, vol. 16, no. 2, pp. 730-743, Feb. 2017. Article (CrossRef Link).

[4] A. Abbasi and M. Ghaderi, "Online algorithms for energy cost minimization in cellular networks," in Proc. of 2014 IEEE 22nd International Symposium of Quality of Service (IWQoS), Hong Kong, pp. 302-307, 2014. Article (CrossRef Link).

[5] S. Bu, F. R. Yu, Y. Cai and X. P. Liu, "When the Smart Grid Meets Energy-Efficient Communications: Green Wireless Cellular Networks Powered by the Smart Grid," IEEE Transactions on Wireless Communications, vol. 11, no. 8, pp. 3014-3024, Aug. 2012. Article (CrossRef Link).

[6] X. Chai; Z. Zhang; K. Long, "Joint Spectrum-Sharing and Base Station Sleep Model for Improving Energy Efficiency of Heterogeneous Networks," IEEE Systems Journal, vol.PP, no.99, pp.1-11. Article (CrossRef Link).

[7] Han, Guangjie, et al. "Cross-layer optimized routing in wireless sensor networks with duty cycle and energy harvesting," Wireless Communications \& Mobile Computing, vol. 15, Issue 16, pp. 1957-1981, 2015. Article (CrossRef Link).

[8] H. Zhang; S. Huang; C. Jiang; K. Long; V. C. M. Leung; H. V. Poor, "Energy Efficient User Association and Power Allocation in Millimeter Wave Based Ultra Dense Networks with Energy Harvesting Base Stations," IEEE Journal on Selected Areas in Communications , vol. 35, no. 9, pp. 1936-1947, 2017. Article (CrossRef Link).

[9] Dong, Y., et al, "Full Duplex Distributed Antenna Systems With Energy Harvesting," in Proc. of IEEE Global Communications Conference. IEEE, 2015. Article (CrossRef Link).

[10] H. Zhang, J. Du, J. Cheng and V. C. M. Leung, "Resource Allocation in SWIPT Enabled Heterogeneous Cloud Small Cell Networks with Incomplete CSI," 2016 IEEE Global Communications Conference (GLOBECOM), Washington, DC, pp. 1-5, 2016.

[11] L. Yu, T. Jiang and Y. Cao, "Energy Cost Minimization for Distributed Internet Data Centers in Smart Microgrids Considering Power Outages," IEEE Transactions on Parallel and Distributed Systems, vol. 26, no. 1, pp. 120-130, Jan. 2015. Article (CrossRef Link).

[12] Chenrui Jin, Xiang Sheng and P. Ghosh, "Energy efficient algorithms for Electric Vehicle charging with intermittent renewable energy sources," 2013 IEEE Power \& Energy Society General Meeting, Vancouver, BC, pp. 1-5, 2013. Article (CrossRef Link).

[13] Josip, Lorincz, G. Tonko, and P. Goran. "Measurements and Modelling of Base Station Power Consumption under Real Traffic Loads," Sensors, 12(4), 4181-4310, 2012.

Article (CrossRef Link).

[14] Michael Neely, "Stochastic Network Optimization with Application to Communication and Queueing Systems," Stochastic Network Optimization with Application to Communication and Queueing Systems, 1, Morgan \& Claypool, pp. 1-211, 2010. Article (CrossRef Link).

[15] Y. Guo, Y. Fang and P. P. Khargonekar, "Optimal Workload and Energy Storage Management for Cloud Data Centers," in Proc. of MILCOM 2013 - 2013 IEEE Military Communications Conference, San Diego, CA, pp. 1850-1855, 2013. Article (CrossRef Link).

[16] M. J. Neely, A. Saber Tehrani and A. G. Dimakis, "Efficient Algorithms for Renewable Energy Allocation to Delay Tolerant Consumers," in Proc. of 2010 First IEEE International Conference on Smart Grid Communications, Gaithersburg, MD, pp. 549-554, 2010. Article (CrossRef Link).

[17] C. Jin, X. Sheng and P. Ghosh, "Optimized Electric Vehicle Charging With Intermittent 
Renewable Energy Sources," IEEE Journal of Selected Topics in Signal Processing, vol. 8, no. 6, pp. 1063-1072, Dec. 2014. Article (CrossRef Link).

[18] Western Wind resources Dataset. The national renewable energy laboratory. Article (CrossRef Link).

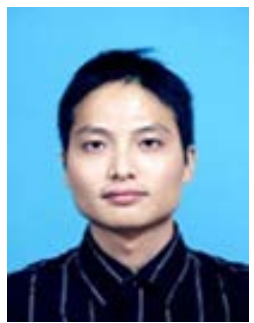

Qingyong Deng was born in Hunan province, P.R.China. He received the B.Eng. degree in Electrical Information Engineering from Xiangtan University in 2004, the M.Eng. degree in Signal and Information Processing from Xiangtan University in 2009.He is currently a Ph.D candidate in Beijing University of Posts and Telecommunications. From 2004 to now, he is a teacher at the college of Information Engineering, Xiangtan University. His research interests include wireless communication and IOT. His current researches focus on cognitive network, machine-to-machine communication, smart grid, and energy harvesting communication.

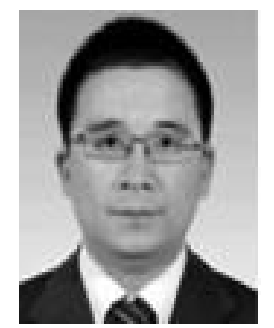

Xueming Li received his B.S. degree from the University of Science and Technology of China (USTC) in 1992 and Ph.D. degree from the Beijing University of Posts and Telecommunications (BUPT) in 1997, all in electronic engineering. From 1997 to 1999, he was a post-doctor researcher in Institute of Information Science of Beijing Jiaotong University (BJTU). He has been working for BUPT since 1999. In 2002, he once worked as a guest lecturer in the Karlsruhe University, Germany. His current research interests include machine learning, digital image processing and multimedia telecommunication. Up to now, he has published more than 40 papers in the field of signal and information processing. Li now is a senior member of Chinese Institute of Electronics, and a senior member of China Society of Image and Graphics.

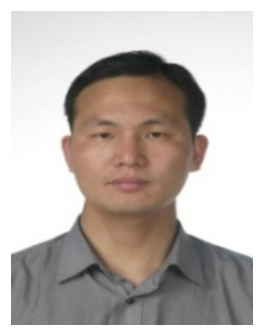

Zhetao Li was born in Hunan province, P.R.China. He received the B.Eng. degree in Electrical Information Engineering from Xiangtan University in 2002, the M.Eng. degree in Pattern Recognition and Intelligent System from Beihang University in 2005, and the Ph.D. degree in Computer Application Technology from Hunan University in 2010. From 2010 to now, Dr. Li is a professor in College of Information Engineering, Xiangtan University. From Dec 2013 to Dec 2014, he was a post-doc in wireless network at Stony Brook University. From Dec 2014 to Dec 2015, he was an invited professor at Ajou University.He has published about thirty papers and submitted about forty patents on wireless network and compressive sensing in the past five years. He was a lead guest editor of two special issues of PPAN.For his successes in teaching and research he received the Second Prize of Fok Ying Tung Education Foundation Fourteenth Young Teachers Award in 2014.His research interests include wireless communication and signal processing. His current researches focus on cognitive network, the 4G mobile communication networks, compressive sensing, and distributed data collection. 


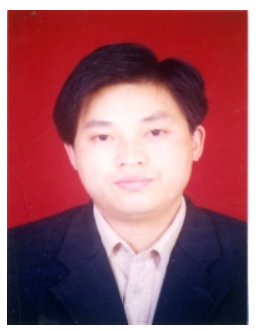

Anfeng Liu is a professor of School of Information Science and Engineering of Central South University, China. He is also a Member (E200012141 M) of China Computer Federation (CCF). He received his M.Sc. and Ph.D. degrees from Central South University, China, in 2002 and 2005, both in computer science. His major research interest is wireless sensor networks.

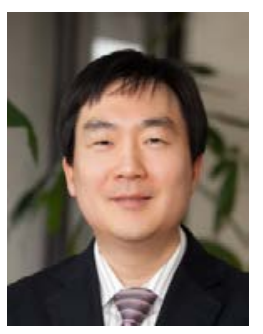

Young-June Choi (S'02-M'08-SM'13) received the B.S., M.S., and Ph.D. degrees from the Department of Electrical Engineering and Computer Science, Seoul National University, in 2000, 2002, and 2006, respectively. From 2006 to 2007, he was a Post-Doctoral Researcher with the University of Michigan, Ann Arbor, MI, USA. From 2007 to 2009, he was with NEC Laboratories America, Inc.,Princeton, NJ, USA, as a Research Staff Member. He is currently an Associate Professor with Ajou University, Suwon, South Korea. His research interests include beyond mobile wireless networks, radio resource management, and cognitive radio networks. He was a recipient of the Gold Prize at the Samsung Humantech Thesis Contest in 2006, Haedong Young Researcher Award in 2015, and Best Paper Award from Journal of Communications and Networks in 2015. 